\title{
Amniotic Umbilical Cord Particulate for Discogenic Pain
}

Derek Buck, MD, DC

Financial Disclosures:

None reported.

Support: None reported.

Address correspondence to Derek Buck, MD, DC, Sideline

Orthopedics and Sports Medicine $250 \mathrm{~S}$ Main St, Ste 224 A, Blacksburg, VA 24060-
Context: Discogenic low back pain is the most common type of low back pain and is a major cause of morbidity worldwide. Current nonoperative treatment options are limited in efficacy and lack evidence of long-term pain relief; thus, there is an unmet clinical need for an effective treatment for patients with discogenic pain. Amniotic membrane and umbilical cord (AMUC) particulate may be useful in relieving pain and inhibiting the degenerative cascade in patients with discogenic pain by reducing inflammation.

Objective: To evaluate the effectiveness of AMUC particulate for discogenic pain.

Methods: Six months of conservative therapy failed in patients who had discogenic pain confirmed by magnetic resonance imaging and provocative discography. They subsequently received intradiskal injection of 50 to $100 \mathrm{mg}$ of AMUC particulate for cervical, lumbar, and lumbosacral disks.

Results: A total of 11 patients with 20 disks were included. Before treatment, all patients reported severe pain, and 10 patients took opioids daily. After treatment, the median reported pain relief was $40 \%, 50 \%$, and $75 \%$ at 1 -month $(n=6), 3$ months $(\mathrm{n}=8)$, and 6 months $(\mathrm{n}=5)$, respectively. Complete pain relief was noted in 1 patient; however, 2 patients (18\%) reported no pain relief at 1 and 3 months. No adverse events, repeated procedures, or complications occurred.

Conclusion: This preliminary evidence suggests that a single intradiskal injection of AMUC particulate is safe and may provide symptomatic pain relief in some patients with discogenic pain.

J Am Osteopath Assoc. 2019;119(12):814-819

doi:10.7556/jaoa.2019.138

Keywords: amniotic tissue, back pain, discogenic pain, umbilical cord

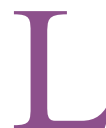
ower back pain affects many people and causes a substantial socioeconomic burden on society, with estimated health care and indirect costs at 100 billion dollars annually. ${ }^{1}$ Potential causes of lower back pain include degenerative spine conditions, such as facet joint disease, spinal stenosis, disk herniations, spondylolysis, or discogenic pain. ${ }^{2}$ According to one study, up to $39 \%$ of all lower back pain is believed to be discogenic in origin. ${ }^{3}$ Discogenic pain is pain originating from a damaged vertebral disk and be caused by inflammation, dehydration of the nucleus pulposus, decreased disk height, annular tears, and impaired mechanical function of the disk. ${ }^{1}$ The criterion standard for diagnosing discogenic pain remains provocative discography whereby contrast is injected into a disk, and concordant pain is reproduced with increasing intradiskal pressure. ${ }^{1}$ 
Nonoperative treatment for patients with discogenic pain may include traction, steroid therapy, methylene blue injection, and ablative therapy. However, few high-quality studies have evaluated these treatments for reducing discogenic pain in the lower back. ${ }^{4}$ In addition, 6 of 11 randomized clinical trials failed to detect significant differences between active and placebo therapies, and it is unclear whether these interventions conferred stable long-term benefit. ${ }^{1}$ Hence, there remains an unmet treatment need for patients with discogenic pain, and thus, these patients are left with significant pain and disability.

Human amniotic membrane and umbilical cord (AMUC) allografts have been used clinically for more than 100 years and have a long history of safe use in a variety of clinical applications, including ophthalmology, sports medicine, and wound care because of their known anti-inflammatory, antiscarring, and proregenerative properties. ${ }^{5}$ Clinically observed effects after injection of AMUC particulate include reduction of pain, accelerated wound healing without scar formation, and quicker return to functionality depending on the indication. ${ }^{6-11}$ In particular, AMUC has also been shown to reduce pain and improve quality of life in prospective, controlled, randomized clinical trials of patients undergoing lumbar microdiscectomy surgery ${ }^{12}$ and also shown to reduce pain and opioid consumption in patients with facet joint syndrome. ${ }^{13}$ Because of these beneficial properties, AMUC particulate may be useful in relieving pain and inhibiting the degenerative cascade in patients with discogenic pain by reducing inflammation. ${ }^{14}$ The purpose of this case series is to report the use of AMUC particulate matrix in patients presenting with discogenic back pain.

\section{Methods}

Patient's medical records were reviewed by D.B. if they underwent intradiskal AMUC injection for discogenic pain between January and November 2018. The inclusion criteria were age 18 to 85 years and at least 1 month of follow-up data. Before AMUC treatments, patients had attempted conservative treatment (eg, physical therapy, oral nonsteroidal anti-inflammatory drugs, ice, and behavioral therapy) for 6 months without any pain resolution. Owing to the refractory nature, additional treatments were discussed with the patients, including radiofrequency ablation, spinal cord stimulators, intradiskal or epidural steroid injections, and AMUC injection. After discussing the risks and benefits, each patient decided on AMUC.

Diagnosis of discogenic pain was routinely performed as follows: patient history was collected and focused on usual pain encounters, previous diagnostic and therapeutic interventions, medical history, and allergies. Physical examination revealed that the patients' pain was exacerbated by activity and relieved by rest; the pain was primarily felt in the axial distribution without apparent radicular pain of the lower extremity. Magnetic resonance imaging confirmed a high-intensity zone on T2-weighted imaging with at least $50 \%$ of disk height remaining. It also confirmed that there was no significant disk protrusion, severe lumbar spinal stenosis, or tumors. Provocative discography confirmed positive per Spine Intervention Society guidelines (ie, concordant pain response of $\geq 7 / 10$, symptoms at $<50$ psi above opening pressure, volume limit of $3.5 \mathrm{~mL}$, and at least grade 3 annular tear per Dallas classification, ie, computed tomographic confirmation of contrast spread to at least the outer one-third annulus). Patients were never sedated for any diagnostic procedure as to not invalidate the study.

Prior to injection, patients received intravenous antibiotics. Patients were then placed in a prone position with their head turned to one side and a pillow under the lower abdomen above the iliac crest to reduce lumbar lordosis. Patients were asked to rotate the inferior aspect of the pelvis anteriorly toward the table to tip the iliac crests posteriorly. Skin was cleansed with povidone iodine to reduce the risk of infection or diskitis. The C-arm was rotated and centered on the appropriate disk. The intradiskal injection involved a posterolateral approach with use of a coaxial device 
(88.9-mm 18-gauge spinal needle and 198-mm 22-gauge disk needle). During incremental progression of the needle, needle-tip positioning and orientation in lateral and frontal plans were assessed by sequential fluoroscopic imaging. Although cervical disks have similar innervation as lumbar disks, cervical nuclei pulposi are located more posteriorly than lumbar disk nuclei pulposi, and the needle was positioned accordingly. Patients received a single injection of $1 \mathrm{~mL}$ of iopamidol 300 contrast and AMUC particulate with 2 $\mathrm{mL}$ of saline in the nuclei pulposi (observed at both lateral and anteroposterior regions of the affected disk). The final total injection volume of saline was determined based on intradiskal pressure and integrity of the disk determined by assessing the amount of pressure it could hold. No anesthetic was injected into the intervertebral disk. After injection, the site was wrapped with a sterile bandage and the patient taken to the recovery room and observed for at least 30 minutes. Patients were instructed to ice the area for relief as needed for approximately 48 hours and advised that pain and discomfort may be felt during the first 36 to 72 hours. Patients could immediately return to normal activities. They were called every week and asked to return at 1, 3, and 6 months. At each follow-up visit, patients were asked to report their perceived level of pain relief, with $0 \%$ being the index pain at initial consult and $100 \%$ being complete pain relief. In addition, safety was assessed by complications and adverse events considered related to the product and procedure.

The AMUC particulate used in this study has been commercially available since 2013 in the United States as a 361 human cell- and tissue-based product. The AMUC particulate is derived from donated human placental tissue following healthy, live, caesarian delivery, full-term births, and then stored at low temperatures. ${ }^{15}$ During processing, the placenta is cleaned of blood with phosphate-buffered saline under aseptic conditions prior to separation of amniotic membrane and umbilical cord by blunt dissection. The amniotic membrane and umbilical cord are micronized, lyophilized, and termin- ally sterilized. The AMUC particulate comes as a dry powder in a small vial stored at room temperature.

\section{Results}

A total of 11 patients ( 6 female, 5 male) received a single intradiskal injection of AMUC particulate for single-level $(n=4)$ and multilevel $(n=7)$ discogenic pain, with a total of 20 disks treated. Nine patients received only lumbar or lumbosacral intervertebral disk injection, 1 patient received only cervical disk injection, and 1 patient received both lumbar and cervical disk injection. Prior to AMUC injection, all patients reported severe pain despite opiate use in 10 of the 11 patients $(>100 \mathrm{mg}$ morphine equivalents per day). Each disk received a median dose level of 100 mg of AMUC particulate (range, 50-100 mg): 9 disks received $100 \mathrm{mg}$ of $\mathrm{AMUC}$, and 11 disks received 50 mg of AMUC particulate (Table). The Figure shows injection of AMUC particulate into L5-S1 in patient 1.

After the AMUC injection, patients reported mild discomfort for 2 weeks. By 3 weeks, patients reported rapid improvement. The median reported pain relief was $40 \%, 50 \%$, and $75 \%$ at 1 month ( $\mathrm{n}=6), 3$ months $(\mathrm{n}=8)$, and 6 months $(\mathrm{n}=5)$, respectively. When considering the last follow-up visit for each patient (mean [SD], 4.1 [1.9] months), the median reported pain relief was $75 \%$. Two patients (18\%) reported no pain relief at the 1- and 3-month follow-up visits. Both patients were administered $100 \mathrm{mg}$ of AMUC particulate in the lower lumbar regions.

No adverse events or complications (eg, diskitis, neural injury, bleeding, bruising, myelopathy, or epidural abscess) occurred over the 6-month follow-up period.

\section{Discussion}

The invertebrate disk is composed of the outer annulus fibrosis (formed by fibrocartilage) and the inner nucleus pulposus (formed by collagens, proteoglycans, and water) sandwiched inferiorly and superiorly by cartilage end plates. ${ }^{16}$ Discogenic pain originates from 


\section{Table.}

Pain Relief Status in Patients Receiving Amniotic Umbilical Cord Particulate for Discogenic Pain

\begin{tabular}{|c|c|c|c|c|c|c|}
\hline \multirow[b]{2}{*}{ Patient } & \multirow[b]{2}{*}{ Gender } & \multirow[b]{2}{*}{ AMUC Dose Per Level, mg } & \multirow[b]{2}{*}{ Location } & \multicolumn{3}{|c|}{ Pain Relief, \% ${ }^{a}$} \\
\hline & & & & $1 \mathrm{mo}$ & 3 mo & $6 \mathrm{mo}$ \\
\hline 1 & M & 50 & L3/L4, L4/L5, L5/S1 & NA & NA & 75 \\
\hline 2 & M & 100 & L4/L5 & NA & NA & 75 \\
\hline 3 & $\mathrm{~F}$ & 100 & L4/L5 & 0 & 0 & 0 \\
\hline 4 & M & 100 & L5/S1 & 0 & 0 & NA \\
\hline 5 & $\mathrm{~F}$ & 50 & $\mathrm{C} 4 / \mathrm{C} 5, \mathrm{C} 6 / \mathrm{C} 7$ & NA & 40 & 75 \\
\hline 6 & $\mathrm{~F}$ & 50 & L4/L5, L5/S1 & 50 & 50 & NA \\
\hline \multirow[t]{2}{*}{7} & M & 100 & L2/L3, L4/L5 & 50 & 50 & 50 \\
\hline & & 50 & $\mathrm{C} 3 / \mathrm{C} 4$ & NA & 60 & 60 \\
\hline 8 & M & 50 & L4/L5, L5/S1 & 100 & NA & NA \\
\hline 9 & $\mathrm{~F}$ & 100 & L4/L5, L5/S1 & 30 & 50 & NA \\
\hline 10 & $\mathrm{~F}$ & 50 & L5/S1 & NA & 80 & NA \\
\hline \multirow[t]{2}{*}{11} & $\mathrm{~F}$ & 100 & L4/L5, L5/S1 & NA & 80 & NA \\
\hline & & & Total, mean (SD) & $38.3(37.6)$ & $45.6(29.2)$ & $55.8(29.2)$ \\
\hline
\end{tabular}

a Pain scale: $0=$ pain at initial consult to $100=$ complete pain relief.

Abbreviations: AMUC, amniotic membrane and umbilical cord; NA, not applicable.

damage to the vertebral disk; however, the pathogenesis is poorly understood. Potential causes include osmotic pressure loss in the nucleus due to aging, which leads to dehydration and degeneration or potential inflammation due to injury. ${ }^{17}$ Studies suggest that growth factors and inflammatory cells play a role in repair, and disks start to form zones of uncharacteristic vascularized granulation tissue with extensive innervation deep into the annulus fibrosus and nucleus pulposus. ${ }^{18}$ Based on this theory, several new treatments have been proposed, including mesenchymal stem cells, bone marrow aspirate, bone marrow aspirate concentrate, exosomes, fibrin glue or platelet-rich plasma to promote regeneration, reduce inflammation, and/or ablate nociceptive nerves in the outer annulus. However, data thus far are limited and require further studies. ${ }^{19-24}$

In the current study, a single intradiskal injection of AMUC particulate was shown to provide a median pain relief of $40 \%$ at 1 month, $50 \%$ at 3 months, and
$75 \%$ at 6 months. Despite the beneficial results observed in this study, 2 patients (patients 3 and 4) reported no symptomatic relief. I was unable to determine the factor that may have hindered the therapeutic benefit. Similarly, Bodor et $\mathrm{al}^{25}$ showed that two-thirds of patients with discogenic pain treated with platelet-rich plasma showed positive outcomes, and $28.8 \%$ of patients treated with platelet leukocyte-rich plasma did not show any improvement despite weekly injections for 6 weeks. $^{26}$ Yet, in a double-blind randomized controlled trial, platelet-rich plasma was shown to significantly improve pain at 8 weeks compared with placebo group. ${ }^{27}$ Hence, regenerative medicine may play a role in discogenic pain. There remains an unmet need for treatment options, as the results of current standard therapy fail to significantly differentiate from placebo therapy. ${ }^{1}$

Although this particular application of AMUC particulate is new, AMUC has been used clinically since 


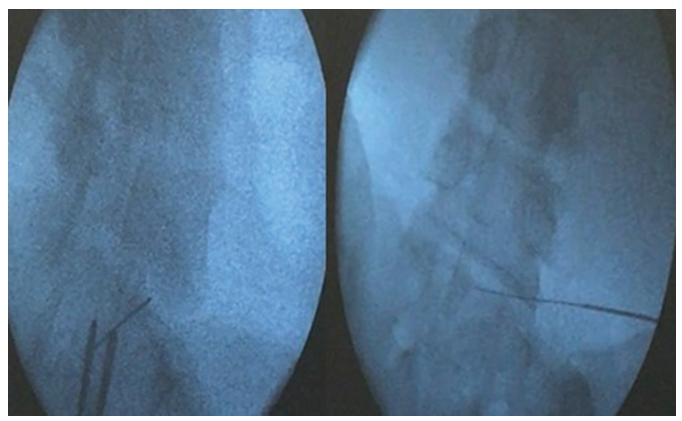

Figure.

Injection of amniotic membrane and umbilical cord particulate into L5-S1 in patient (1) with discogenic pain.

$1910 .^{28}$ The anti-inflammatory properties of the AMUC tissue, which downregulate pro-inflammatory cytokines, induces apoptosis of pro-inflammatory cells and promotes polarization of anti-inflammatory cells. ${ }^{5}$ Preclinical and clinical studies have confirmed these therapeutic benefits in diseased joints, where AMUC particulate was shown to attenuate progressive cartilage degeneration and provide pain relief. ${ }^{9,29}$ Hence, these anti-inflammatory actions hypothetically may have attenuated the inflammatory mediators in the inveterate disk, which led to reduced pain as observed in the current study. Further studies are needed to assess inflammatory biomarkers and determine the optimal dosing regimen. Preclinical studies have shown a dosedependent response ${ }^{29}$; however, no significant differences were found between the 50- and 100-mg AMUC groups in this series, presumably due to small sample size. Hence, larger studies with a longer follow-up duration and validated outcome measures are needed to determine its efficacy in pain reduction for this major cause of lower back pain, especially to determine whether this treatment can serve as a nonopioid alternative.

\section{Conclusion}

Preliminary evidence suggests that a single intradiskal injection of AMUC is safe and may provide symptomatic pain relief in some patients with discogenic pain. Further studies with larger sample sizes are warranted.

\section{References}

1. Lu Y, Guzman JZ, Purmessur D, et al. Nonoperative management for discogenic back pain: a systematic review. Spine (Phila Pa 1976). 2014;39(16):1314-1324. doi:10.1097/BRS.0000000000000401

2. Deyo RA, Weinstein JN. Low back pain. N Engl J Med. 2001;344 (5):363-370.

3. Schwarzer AC, Aprill CN, Derby R, Fortin J, Kine G, Bogduk N. The prevalence and clinical features of internal disk disruption in patients with chronic low back pain. Spine (Phila Pa 1976). 1995;20 (17):1878-1883

4. Muzin S, Isaac Z, Walker JIII. The role of intradiskal steroids in the treatment of discogenic low back pain. Curr Rev Musculoskelet Med. 2008;1(2):103-107. doi:10.1007/s12178-007-9015-y

5. Tseng SC. HC-HA/PTX3 purified from amniotic membrane as novel regenerative matrix: insight into relationship between inflammation and regeneration. Invest Ophthalmol Vis Sci. 2016;57(5):ORSFh1-8. doi:10.1167/iovs.15-17637

6. Hanselman AE, Tidwell JE, Santrock RD. Cryopreserved human amniotic membrane injection for plantar fasciitis: a randomized, controlled, double-blind pilot study. Foot Ankle Int. 2015;36 (2):151-158. doi:10.1177/1071100714552824

7. Warner M, Lasyone L. An open-label, single-center, retrospective study of cryopreserved amniotic membrane and umbilical cord tissue as an adjunct for foot and ankle surgery. Surg Technol Int. 2014;25:251-255.

8. Garras D, Scott R. Particulate umbilical cord/amniotic membrane for the treatment of plantar fasciitis. Foot Ankle Orthopaed. 2017;2 doi: $10.1177 / 24730114175000174$

9. Castellanos R, Tighe S. Injectable amniotic membrane/umbilical cord particulate for knee osteoarthritis: a prospective, single-center pilot study. Pain Med. 2019. pii:pnz143. doi:10.1093/pm/pnz143

10. Bemenderfer TB, Anderson RB, Odum SM, Davis WH. Effects of cryopreserved amniotic membrane-umbilical cord allograft on total ankle arthroplasty wound healing. J Foot Ankle Surg. 2019;58 (1):97-102. doi:10.1053/j.jfas.2018.08.014

11. Covell DJ, Cohen B, Ellington JK, Jones CP, Davis WH, Anderson RB. The use of cryo-preserved umbilical cord plus amniotic membrane tissues in the resection of tarsal coalition. Foot Ankle Orthopaed. 2016;1(1). doi:10.1177/2473011416S00311

12. Anderson DG, Popov V, Raines AL, O'Connell J. Cryopreserved amniotic membrane improves clinical outcomes following microdiscectomy. Clin Spine Surg. 2017;30(9):413-418. doi:10.1097/ BSD. 0000000000000544

13. Bennett DS. Cryopreserved amniotic membrane and umbilical cord particulate for managing pain caused by facet joint syndrome: a case series. Medicine (Baltimore). 2019;98(10):e14745. doi:10.1097/ MD. 0000000000014745

14. Navone SE, Marfia G, Giannoni A, et al. Inflammatory mediators and signalling pathways controlling intervertebral disk degeneration. Histol Histopathol. 2017;32(6):523-542. doi:10.14670/HH-11-846

15. Cooke M, Tan EK, Mandrycky C, He H, O'Connell J, Tseng SC Comparison of cryopreserved amniotic membrane and umbilical cord tissue with dehydrated amniotic membrane/chorion tissue. J WoundCare. 2014;23(10):465-474, 476. doi:10.12968/ jowc.2014.23.10.465 
16. Raj PP. Intervertebral disc: anatomy-physiology-pathophysiologytreatment. Pain Pract. 2008;8(1):18-44. doi:10.1111/ j.1533-2500.2007.00171.x

17. Peng BG. Pathophysiology, diagnosis, and treatment of discogenic low back pain. World J Orthop. 2013;4(2):42-52. doi:10.5312/wjo.v4. i2.42

18. Peng B, Wu W, Hou S, Li P, Zhang C, Yang Y. The pathogenesis of discogenic low back pain. J Bone Joint Surg Br. 2005;87:62-67.

19. Sanapati J, Manchikanti L, Atluri S, et al. Do regenerative medicine therapies provide long-term relief in chronic low back pain: a systematic review and metaanalysis. Pain Physician. 2018;21 (6):515-540.

20. Navani A, Manchikanti L, Albers SL, et al. Responsible, safe, and effective use of biologics in the management of low back pain American Society of Interventional Pain Physicians (ASIPP) guidelines. Pain Physician. 2019;22:S1-S74.

21. Zeckser J, Wolff M, Tucker J, Goodwin J. Multipotent mesenchymal stem cell treatment for discogenic low back pain and disk degeneration. Stem Cells Int. 2016;2016:3908389. doi:10.1155/2016/ 3908389

22. Yin W, Pauza K, Olan WJ, Doerzbacher JF, Thorne KJ. Intradiscal injection of fibrin sealant for the treatment of symptomatic lumbar internal disk disruption: results of a prospective multicenter pilot study with 24-month follow-up. Pain Med. 2014;15:16-31. doi:10.1111/ pme.12249
23. Pettine K, Dordevic M, Hasz M. Reducing lumbar discogenic back pain and disability with intradiskal injection of bone marrow concentrate: 5-year follow-up. Am J Stem Cell Res. 2018;2:1-4. doi:10.5923/j.ajscr.20180201.01

24. Hansraj KK. Stem cells in spine surgery. Surg Technol Int. 2016;29:348-358.

25. Bodor M, Toy A, Aufiero D. Disc regeneration with platelets and growth factors. In: Lana J, Andrade Santana M, Dias Belangero W, Malheiros Luzo A, eds. Platelet-Rich Plasma. Berlin, Germany: Springer; 2014:265-279.

26. Hussein $M$ and Hussein $T$. Effect of autologous platelet leukocyte rich plasma injections on atrophied lumbar multifidus muscle in low back pain patients with monosegmental degenerative disk disease. SICOT J. 2016;2:12.

27. Tuakli-Wosornu YA, Terry A, Boachie-Adjei K, et al. Lumbar intradiskal platelet-rich plasma (PRP) injections: a prospective, double-blind, randomized controlled study. PM R. 2016;8:1-10. doi:10.1016/j. pmrj.2015.08.010

28. Davis JW. Skin transplantation with a review of 550 cases at the Johns Hopkins Hospital. Johns Hopkins Med J. 1910;15:307-396.

29. Raines AL, Shih MS, Chua L, Su CW, Tseng SC, O'Connell J. Efficacy of particulate amniotic membrane and umbilical cord tissues in attenuating cartilage destruction in an osteoarthritis model. Tissue Eng Part A. 2017;23(1-2):12-19. doi:10.1089/ten.TEA.2016.0088

() 2019 American Osteopathic Association

\section{@TheJAOA on Twitter}

Follow us at http://www.twitter.com/TheJAOA to get involved and stay connected with updates, highlights, and conversations about osteopathic medical research. 